\title{
The ISDF Framework: Towards Secure Software Development
}

\author{
Abdulaziz Alkussayer* and William H. Allen*
}

\begin{abstract}
The rapid growth of communication and globalization has changed the software engineering process. Security has become a crucial component of any software system. However, software developers often lack the knowledge and skills needed to develop secure software. Clearly, the creation of secure software requires more than simply mandating the use of a secure software development lifecycle; the components produced by each stage of the lifecycle must be correctly implemented for the resulting system to achieve its intended goals. This study demonstrates that a more effective approach to the development of secure software can result from the integration of carefully selected security patterns into appropriate stages of the software development lifecycle to ensure that security designs are correctly implemented. The goal of this study is to provide developers with an Integrated Security Development Framework (ISDF) that can assist them in building more secure software.
\end{abstract}

Keywords—Security Patterns, Secure Development, Security Framework

\section{INTRODUCTION}

Until recently, security in software development was viewed as a patch deployed to solve security breaches, or sometimes as an enhancement to an already completed software package. As a result, security considerations were located towards the end of the development lifecycle; particularly as add-on mechanisms and techniques before the system was deployed at the client's premises. Security issues were often raised only after some undetected vulnerability had been compromised. It was not yet understood that developing secure software requires a careful injection of security considerations into each stage of the software development lifecycle [1-4]. However, once the importance of designed-in security was recognized, attention was directed towards improving the development process by considering security as a requirement instead of a corrective measure.

The inspiration for the proposed Integrated Security Development Framework (ISDF) [5, 6] came from recognizing the existence of two common software development pitfalls: i) security is often only an afterthought in software development; ii) many security breaches exploit wellknown security problems. The first issue can only be corrected by mandating the use of a secure development lifecycle to incorporate security considerations across all software development stages. The second must be solved by ensuring that software developers make use of proven development techniques that avoid insecure coding practices.

※ This work is supported by the Institute of Public Administration (IPA) in Saudi Arabia.

Manuscript received December 16, 2009; revised February 23, 2010; accepted March 4, 2010.

Corresponding Author: Abdulaziz Alkussayer

* Dept. of Computer Science, Florida Institute of Technology, Melbourne, Florida, USA (\{alkussaa, wallen\}@my.fit.edu) 


\subsection{Scope}

This study explores how the ISDF framework can be used to integrate the two independent security solutions mentioned above. First, it describes how the ISDF framework incorporates the best features of existing secure SDLCs. Then, it explains a four-stage process for employing security patterns during the development lifecycle. Finally, the study presents a practical example that illustrates the benefits of using the ISDF framework during software development and shows that the combined approach consolidates the secure development best practices that are incorporated into a secure SDLC with the security knowledge built into security patterns.

Fortunately, software security engineering has matured in recent years. Software developers have become more conscious of the fact that security has to be built within the system rather than added onto the system $[4,7,8]$. Thus, software security research has been active in two areas: improving engineering best-practices and increasing the use of security knowledge during development.

To address the first area, significant work has been done to formulate a methodology that considers security throughout the Secure Software Development Lifecycle (SDLC). The objective is to provide a set of development guidelines and rules on how to build more secure software. Among the many advantages of such methodologies is the ability to equip software developers with easy-to-follow security guidelines. These methodologies represent the best known engineering practices for building secure software. Three well-known approaches are the Security Development Lifecycle (SDL) [9], the Comprehensive Lightweight Application Security Process (CLASP) [10] and the Software Security TouchPoints [11]. A recent discussion of bestpractices distilled from these approaches and a few others can be found in the Fundamental Secure Software Development initiative by SAFECode ${ }^{1}$ [12].

It has recently been recognized that security knowledge may be encapsulated within security patterns. A pattern describes a time-tested generic solution to a recurring problem within a specific context [13]. Since 1977 when patterns were first introduced by Alexander et al. [14], they have become a very popular method of encapsulating knowledge in many disciplines. In software engineering, design patterns and security patterns have gained significant attention from the research community. Moreover, software design patterns have become increasingly popular since publication of the Gang-of-Four (GoF) book [15]. Although design patterns have been widely adopted in most of today's development libraries and programming tools, the use of security patterns is more recent. They gained popularity following the seminal work by Yoder and Barcalow [16], which presented seven architectural patterns that are useful in developing the security aspects of a system and used natural language and (GoF) templates to describe their patterns. Since then, many other security patterns have been published. Although many of the published security patterns are considered to be merely guidelines or principals [17], security patterns have proven to be effective methods of dealing with security problems in a software system. Nevertheless, significant effort and security expertise are needed to properly apply them to a real software development situation.

\subsection{Contribution}

The main contribution of this study is to show by example how the ISDF framework can be

1 SAFECode is a global industry-led effort to identify and promote best-practices for developing and delivering more secure software and hardware services. 
used to integrate security patterns published in the literature into a secure software development process. While many research efforts focus on applying security controls at latter stages (e.g. coding and testing) of the development process [18-22], the focus of this work is to guide the development process at earlier stages (e.g. requirements and design) by providing developers with a more systematic approach. To an extent, significant effort and security expertise are needed to properly apply the correct set of patterns to a real software development situation.

Nevertheless, the hope is that as the process explained in the framework is improved through industrial application in different contexts, developers will learn to use and integrate patterns into new development situations more effectively. Moreover, as the security patterns literature matures and pattern templates include specifics on when or how to apply them, the level of security knowledge required to adopt the framework will decrease dramatically.

The rest of this paper is structured as follows. Section 2 provides a brief overview of secure software development and security patterns. Section 3 describes the ISDF framework and presents a practical example that illustrates the use of the ISDF framework to effectively develop more secure software. Section 4 provides an overview of related work. Section 5 contains our conclusions and a brief discussion of future work.

\section{BACKGROUND}

In this section, a survey of the current state of secure development practices and security patterns is presented.

\subsection{Secure Software Development}

In recent literature, a number of approaches are discussed for developing secure software. The Fundamental Secure Software Development guide by SAFECode [12] presents the state-of-thepractice development activities for developing more secure software. It encompasses a six-phase software development cycle and discusses the best industry practices required during each phase to produce more secure software. The development phases are: requirements, design, programming, testing, code integrity and handling, and documentation [12]. This guide serves as the main source of the security best practices that are incorporated into our framework.

Three other well-documented secure development methodologies are Microsoft's SDL, which first appeared as a result of the Trustworthy Computing Initiative in 2002 [9], OWASP's CLASP [10] and Software Security TouchPoints, which was proposed by Gary McGraw in 2004 [11]. Although there are observable differences between these methodologies [23], they agree on three key points $[7,8,10,12]$ :

1.Advocate security education.

2.Risk management is essential.

3.Utilization of best practices is crucial.

Microsoft's SDL is based on thirteen stages, spanning the entire development lifecycle [7]. These stages are:

- education and awareness 
- project inception

- defining and following design best practices

- product risk assessment

- risk analysis

- creating security documents, tools and best-practices for customers

- secure coding policies

- secure testing policies

- the security push

- the final security review

- security response planning

- product release

- security response execution.

The software development artifacts mandated by Microsoft's SDL methodology are: requirements, design, implementation, verification, release, and support \& services [7].

CLASP is a well-organized and structured approach for building secure software that is managed and maintained by OWASP [10]. The primary goal of CLASP is to support the construction of software in which security takes a central role. It advocates moving security concerns into the early stages of the software development lifecycle through the use of a prescriptive approach, documenting activities that organizations should be doing. The CLASP process is presented through five high-level perspectives called CLASP Views. These views are broken down into activities, which in turn contain process components. It includes a set of twenty-four highlevel activities and additional resources, and provides a wealth of security resources that make implementing those activities reasonable [10]. For instance, a list of 218 known security vulnerabilities is provided as a categorized checklist that can be used during design and code reviews.

The Software Security Touchpoints methodology depends on the following seven best practices [11]:

- code review

- architectural risk analysis

- penetration testing

- risk-based security testing

- abuse cases

- security requirements

- security operations.

It also mandates six development phases: requirements and use cases, architecture and design, test plan, code, tests and test results, and feedback from the field [8].

\subsection{Security Patterns}

Security patterns have become a reliable approach for effectively addressing security considerations during implementation of a software system. The security patterns book by Schumacher et al. [13] includes forty-six patterns; twenty-five of which address security issues during the design phase. Also, the Core Security Patterns book [24] presents twenty-three security patterns 
for J2EE enterprise application. These patterns are organized over four tiers as following: nine patterns for the web tier, seven patterns for the business tier, three patterns for the web services tier, and four patterns for the identity tier. Many of these patterns are well structured and hence the use of UML diagrams to represent such patterns is common. For example, Fernandez and Pan [25] used UML diagrams to illustrate four security patterns: Authorization, Role Based Access Control, Multilevel Security, and File Authorization.

To better understand the above-mentioned UML modeling of security patterns, one can look at examples of security patterns that are presented in [13, 24]. Fig. 1 describes the Authorization pattern [13].

The second example in Fig. 2 illustrates the dynamics of the Secure Logger pattern [24].

There are also several model-based security patterns. Hatebue et al. [26] presented security patterns using the Security Problem Frame, which is based on the Problem Frame pattern [27] and is used to capture and analyze security requirements. Horvath and Dorges [28] use Petri Nets to model patterns for multi-agent systems, such as the Sandbox and Message Secrecy patterns. Supaporn et al. [29], proposed a more formal method by constructing an extended-BNF-based grammar of security requirements from security patterns.

According to a recent survey [17] there are currently a little over two hundred published security patterns. Despite the fact that thirty-five percent of these (so-called) patterns are considered to be merely guidelines or principals [17], security patterns have been proven to be effective methods of dealing with security problems in a software system. Nevertheless, significant effort and security expertise are needed to properly apply them to a real software development situation.

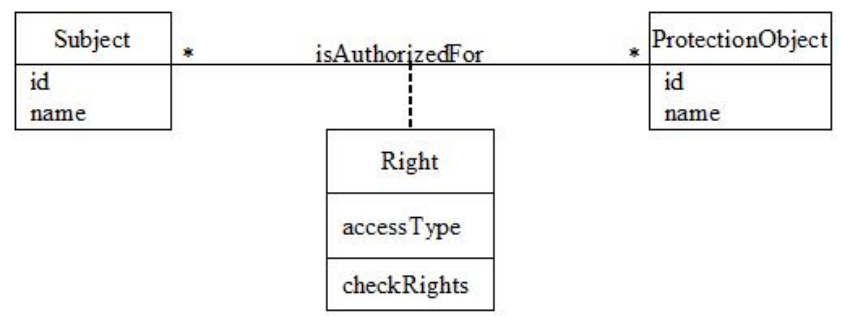

Fig. 1. Authorization Pattern

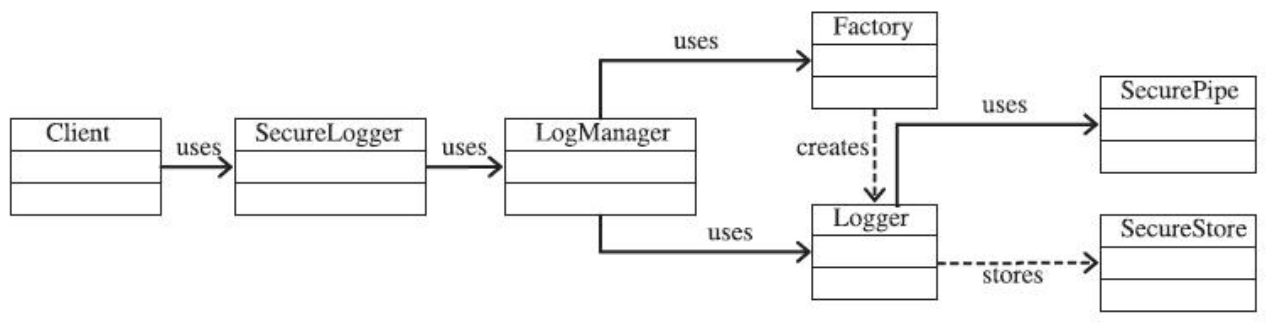

Fig. 2. Secure Logger Pattern 


\section{THE ISDF FRAMEWORK}

The Integrated Security Development Framework (ISDF) consists of two main components, as shown in Fig. 3. The first component is the secure software development best practice, represented on the left-hand side of Fig. 3. The second component is a four-stage security pattern utilization process, which appears on the right-hand side of the figure.

The left hand side of Fig. 3 shows the ISDF mandating a development lifecycle. However, this framework does not represent a particular development lifecycle and hence a conventional development model with six phases is used. These phases are common in any development model. Also, the short activities included in each phase are summarized from [7, 8, 12] collectively. These activities represent the current best engineering practices for developing secure software.

The relation between the best practices activities and security patterns activity is bidirectional. Thus, the key success factor for seamless integration involves interweaving best practices and security pattern activities at every development stage. In the ISDF diagram (Fig. 3) this link is represented by bidirectional arrows at every stage. Next is an explanation of how the framework effectively merges security patterns into the secure software development process.

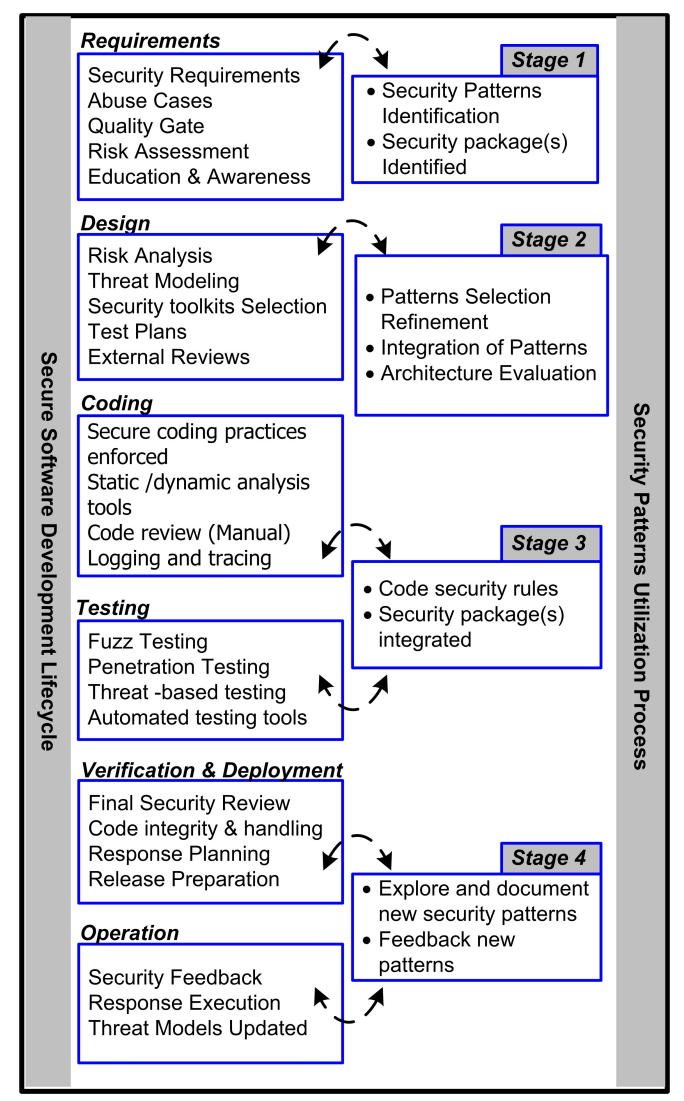

Fig. 3. Integrated Security Development Framework 


\subsection{An eShop Example}

To better illustrate the advantages of the framework, this study uses a simple e-commerce system (called eShop) as a running example. Examples such as this are commonly used because of the popularity and clarity of their prime functionalities. The aim is to demonstrate the effectiveness of the interweaving between best practices and security patterns provided by the framework. Because the focus of this study is on the earlier stages of the development process, this discussion is restricted to the requirements and design stages. Fig. 4 shows a partial architectural design of the eShop using a UML class diagram. Note that, the intent of this diagram is to show some structural components of the eShop system and hence it does not strictly follow the formal UML class diagram notation.

The system, as depicted in Fig. 4, has three external (remotely connected) user groups: customers, product catalog administrators, and customer care representatives. The functional requirements of each user group can be summarized as follows.

- Customers: browse products and place orders.

- Product catalog administrators: remotely manage the product catalog.

- Customer care representatives: remotely manage customers' data and orders.

For the non-functional requirements, this study focuses exclusively on security.

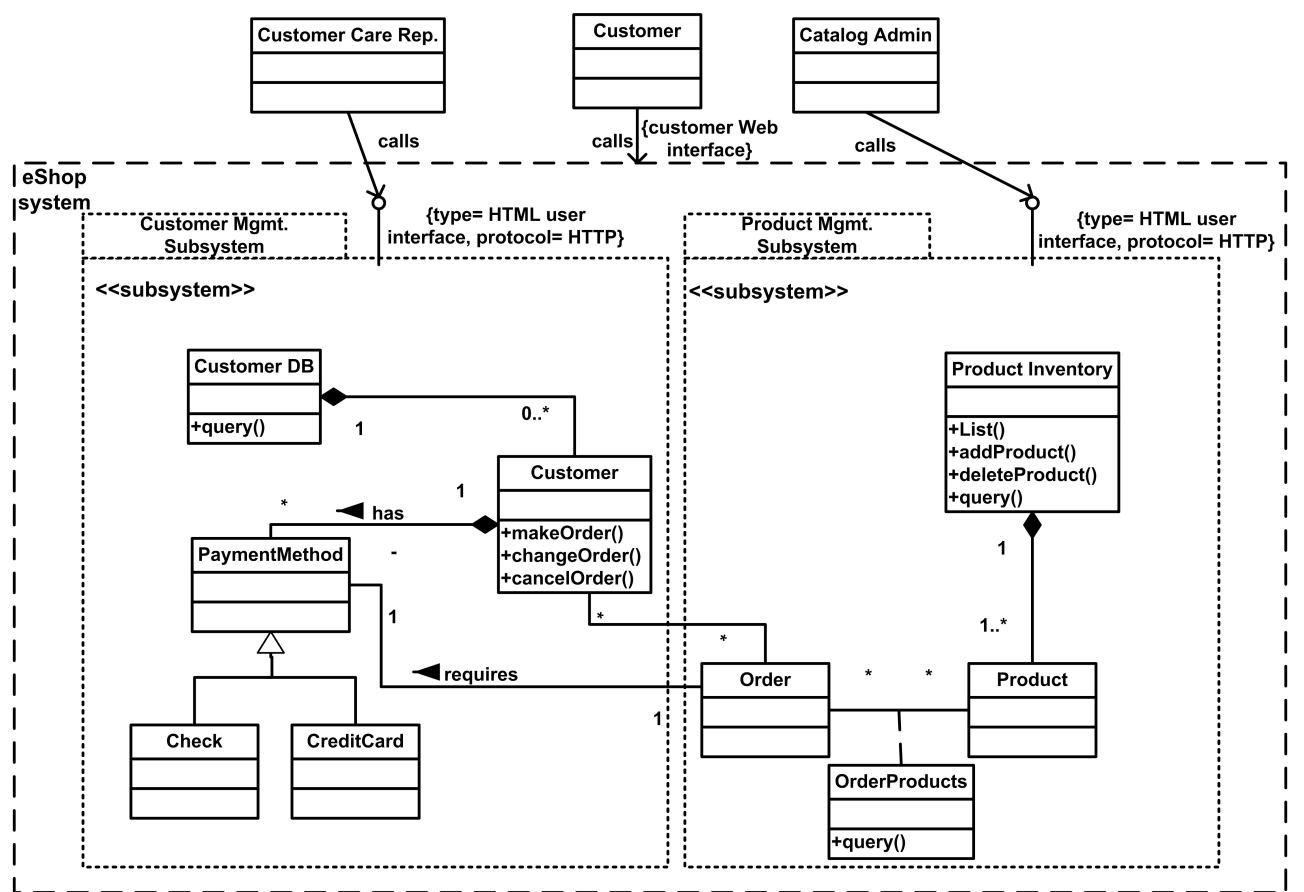

Fig. 4. eShop Preliminary Design 


\subsection{Requirements Stage}

In this stage, security patterns are selected based on the security requirements and on an analysis of potential threats that are determined from the preliminary risk assessment. For example, Access Control, Identification and Authorization patterns [13] can be identified during this stage. Unfortunately, many practitioners unconsciously postpone the decision of identifying and selecting security patterns until the design phase. Since security patterns evolved from design patterns, however, identifying security patterns reveals more than just a design solution. It places security constraints on the system as a whole, as well as on its subcomponents. These security constraints must be rationalized by measurable security requirements and their associated risks must be mitigated. The relationship between security requirements and security patterns is vital. Some researchers $[26,29]$ have investigated this relationship and proposed promising solutions. However, more research is needed in this area.

Moreover, security components (e.g. firewalls) should be identified at this stage in parallel with the corresponding identification of security tools based on best practices. In fact, many practitioners often don't consider security components until the implementation phase. While this delay is somewhat understandable in the sense that the selected security component must be integrated programmatically into the system, the selection of a security component may best be addressed at the requirement stage because subsequent risk assessment and security pattern selection may be affected by this decision. Security pattern repositories, such as [30], and pattern classification and categorization methods, such as [31], can be useful during the pattern selection and identification process.

\section{Stage 1: eShop Requirements}

As mentioned earlier, to better employ the strength of the framework, one must work through the best practice activity and the security pattern activity in parallel fashion. The following is a subset of the security requirements of the eShop.

- Sq1: The system shall enforce authentication of users in a secure manner.

- Sq2: The system shall be able to log and trace back all customer transactions.

- Sq3: The system shall ensure the privacy and protection of customer data and order transactions.

These security requirements explicitly impose the need to satisfy some related security properties. The first requirement requires confidentiality of the access control technique. The second requirement imposes the accountability of customers. The third requirement enforces the confidentiality and integrity of the customer data and transactions.

Now that the security properties of the requirements are clear, security patterns can be identified. For the first requirement, the Authentication Enforcer [24] pattern is selected to handle the problem of how to verify that a subject (customer) is really who they say they are. Next, the accountability property imposes two sub objectives: auditing and non-repudiation [13]. Nonrepudiation focuses on capturing evidence so that users who engage in an event cannot later deny that engagement. Auditing refers to the process of monitoring and analyzing logs to report any indication of a security violation. The Audit Interceptor [24] pattern is selected to intercept and $\log$ requests to satisfy the auditing objective imposed by the second requirement. Finally, 
the Secure Pipe [24] pattern was chosen to fulfill the third requirement and prevent 'man-in-themiddle' attacks.

Note that even though the process of identifying the correct patterns requires a certain level of security expertise, it can be simplified by consulting an organized security patterns inventory, such as [30, 31].

\subsection{Design Stage}

Of course, not all security patterns can be identified as early as the requirement stage. Security patterns may be identified during the design stage to leverage some design constraints. Furthermore, some of the security patterns selected at the previous stage may not adhere to the proposed architecture of the system. Hence, a refinement selection activity should be expected at this stage to resolve such issues.

Schumacher et al. [13] suggests that every security pattern reveals a solution that "consists of a set of interacting roles that can be arranged into multiple concrete design structures". If the structures of security patterns are aligned with the other design structures of the system, the result is an architectural integration between all the structural components.

Although many security design pattern studies have been published, as described in section 2.2, the Open Group presented a very coherent design methodology to improve security [32]. This technical report proposed the use of three generative sequences (one main and two subsequences) for applying security patterns during the design stage. The main sequence is the System Security Sequence and the sub-sequences are the Available System Sequence and the Protected System Sequence [32].

\section{Stage 2: eShop Design}

It is expected that not all security patterns can be discovered as early as the requirements stage. In fact, most patterns identified here are a result of architectural design constraints or as a mitigation strategy to identified threats. Thus, a pattern selection refinement process is crucial in this stage.

It is obvious that with multiple entry points to the system (i.e., an entry point for each user group), some of the patterns identified during the requirement stage may need to be replicated (e.g., the authentication mechanism). As depicted in Fig. 4, three user group entities are interacting with the system at three distinct entry points. However, allowing more entry points may increase the system's risk exposure. For example, a malicious attacker could attempt to impersonate a legitimate user to gain access to his/her resources. This could be particularly serious if the impersonated user has a high level of privilege like a customer care representative role or a product catalog administrator role. The imposter may then be able to compromise the system and disclose customers' credit information or the vendor's financial records.

In addition, an intensive threat modeling process must be utilized during this stage to capture the range of potential threats. Fig. 5 shows a snapshot of the attack tree developed as a part of the threat modeling process for the eShop system.

The design constraints and potential threats identified in this stage collectively influence the refinement process for the preselected patterns. As mentioned above, replicating the authentication mechanism over multiple entry points is problematic and may increase exposure to risks. One possible solution is to unify the system's entry points into a single point of responsibility. 


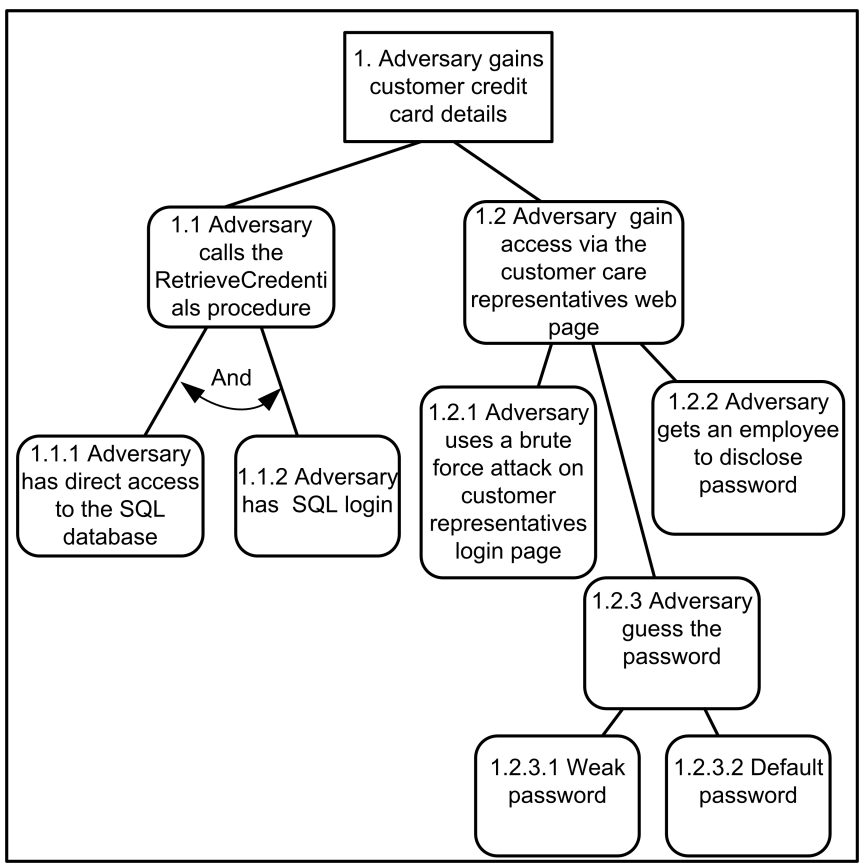

Fig. 5. Partial eShop Attack Tree

This simplifies the control flow since everything must go through a Single Access Point [16]. Fig. 6 depicts a refinement of the selected patterns integrated into the eShop architectural design. As an abstraction to simplify the design, the eShop internal entities are encapsulated in a single entity called eShop inner components.

Along with the Single Access Point, access requests must be validated and authenticated by some type of Check Point [13]. A Check Point establishes a critical section where security must be enforced through a security policy that incorporates different organizational security policies. In terms of the eShop system, the Check Point receives a request from the Single Access Point and provides validation for a user's options within the restrictions of group policy. Then, the Check Point uses a Secure Logger [24] to log the event in a secure manner.

If access is granted, the Check Point then instantiates a Session [24] object for the user. The Session object holds those security variables associated with the user that may be needed by other components. The Check Point uses a Manager component to keep track of active session objects. The eShop inner components entity authorizes the user by asking the Manager for the underlying session object and checking the user's data stored there.

Finally, access to sensitive resources (such as product inventory and customer $D B$ ) may require additional authentication. Therefore, an Authenticator [13] can be used to further verify the identity of the subject prior to granting access to these resources. This places an extra, yet important, defensive shield against malicious attacks like those described earlier. Note that the Authenticator mentioned above is not shown in Fig. 6 due to a simplification of the diagram for the purposes of illustration. 


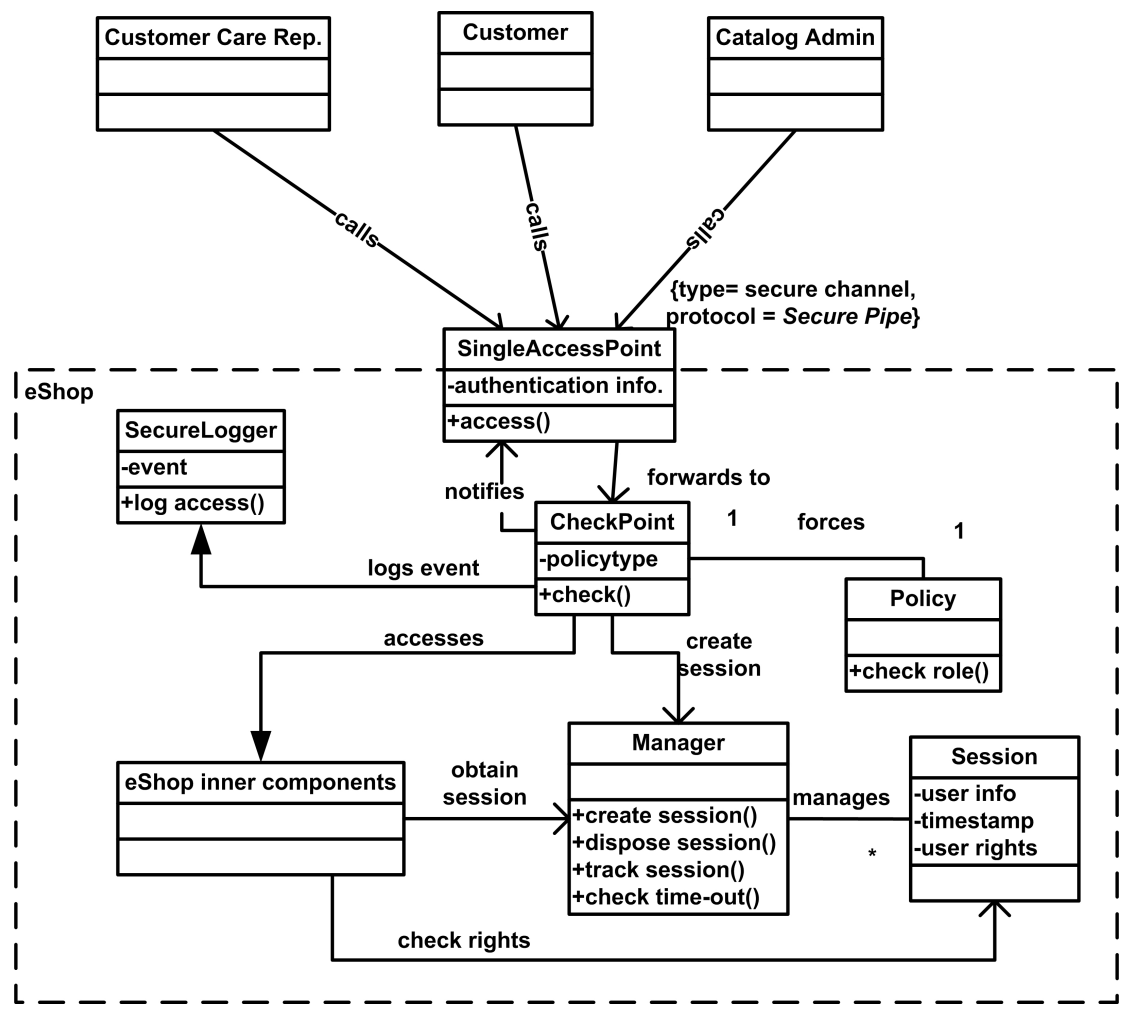

Fig. 6. eShop Integrated Security Patterns

\subsection{Implementation Stage}

In this stage, the security rules produced during the design phase are coded based on the secure coding practices mandated by the secure development lifecycle. The selected security components are integrated with the corresponding system components according to the architectural design. No security patterns exist specifically for this stage [33]. However, many secure development methodologies can use attack patterns [34] as a security education tool and sometimes as test-case drivers.

Also, rigorous threat-based testing for structural components of the pre-selected patterns is fundamental in this stage. Thus, the ISDF anticipates the adherence to the best practices of coding and testing mandated by the secure development lifecycle in the coding and testing phases, respectively.

\subsection{Post Implementation Stage}

This stage corresponds to the last two stages of the secure software development lifecycle in the ISDF, namely deployment and operation. The transition between deployment and operation always raises a critical security concern; carrying the integrity and authenticity of the software source code throughout its chain of custody. The code integrity and handling practice during the deployment phase, as suggested by the SAFECode documentation [12], addresses this concern. 
However, the authors strongly believe that there is a need for a new security pattern to effectively safeguard this transition in parallel with the above mentioned practice.

After the software is deployed into its operational environment, it is important to monitor responses to flaws and vulnerabilities of the system to check for new evolved patterns. Note that it is important to avoid simply declaring that the individual code batches and bug fixes represent new patterns. Once a new security pattern has been found and documented, then that selection should be noted for inclusion in the requirement stage for further security improvement in the subsequent releases.

During the operational lifetime of the system, it is essential to revisit the requirement and design stages before starting to implement the new security countermeasures that resulted from new security threats and attacks. In fact, many recent software security vulnerabilities exist because of the lack of a thorough consideration of the countermeasure defenses implemented at the earlier stages.

\section{RELATED WORK}

Researchers have begun to focus on integrating security patterns into a software development lifecycle. For example, Aprville and Pourzandi [35] investigated the use of security patterns and UMLsec [36] in some phases of a secure software development lifecycle but were hampered by the limited range of patterns available at the time. They also did not describe how patterns could be incorporated into a secure SDL to create a development framework.

Valenzuela [37] recommended a methodology that integrates the ISO 17799 (an international Information Security Standard) with a software development lifecycle. This approach proposed parallel security activities for each stage of the SDL and included a mapping of each stage to the appropriate phase of the ISO 17799 process.

Fernandez et al. [38] proposed a methodology that incorporates security patterns in all development stages of their own lifecycle. Their approach includes guidelines for integrating security from the requirements phase through analysis, design, and implementation. In a more recent paper, Fernandez et al. [39] proposed a combination of three similar methodologies into a single unified approach to build secure systems using patterns, but did not integrate them into an industry-recognized Secure SDL.

Existing studies have focused on using either security patterns or best practices - or a loose combination of the two - to build secure software. However, none has explored the need for a concrete method to incorporate the full strength of the two approaches.

\section{Discussion AND CONCLUSIONS}

This study has presented an integrated framework for developing secure software based on the combination of secure development best practices and security patterns. It has also presented a four-stage software development process to better utilize security patterns within secure development methodologies. Through the case study of the eShop system, this study has shown that the ISDF framework provides a systematic way to assure developing more secure software. In 
particular, it enforces the inclusion of security patterns in the early stages of the secure development process. It has introduced the use of security patterns in the context of development best practice as a general way to control security concerns identified earlier in the secure development process.

The contributions of this study are twofold: first, a general framework that consolidates established security patterns with software development best practices. Combining the two will not only simplify the process of building more secure software, but also reduce the risks associated with using ad-hoc security approaches in software development. Second, the ISDF framework enables developers with limited security experience to more easily and systematically develop secure software. However, the process of selecting and assigning security patterns to individual stages or development situations is not only a domain-specific but also a system-specific design issue, which is not dictated by the framework presented in this paper. This is because a given assignment represents a specific design decision tradeoff and reflects the stakeholders' perspective. The framework, however, can be used to ensure the systematic inclusion of security patterns in the early stages of the development process and promotes their effective use alongside the best practices to build more secure software.

This study helps to bridge the gap between the use of established security patterns and development best practices that exist today. We believe that such a framework not only helps ensure the integration of security patterns with best practices early in the development process, but also helps to resolve two issues noted in the security patterns literature. The first is the observation that 35 percent of the published patterns do not pass the soundness test for patterns and, therefore, are considered to be guidelines or principles rather than formal patterns [17]. For example, security patterns like Asset Valuation and Threat Assessment [13] don't conform to the formal definition of a security pattern $[17,33]$. However, since the ISDF incorporates best practices to guide secure development, patterns such as those can be avoided. The second issue is the lack of patterns for some parts of the development phase (e.g., the small number of attack patterns for use in the design phase) [33]. The framework resolves this limitation by mandating concrete best practices in parallel with security patterns.

A successful verification process for the proposed framework is one that evaluates the output of each constituent subcomponent (stage) of the framework and asserts whether adequate control of security exposure has been achieved or not. However, latter stages (i.e. implementation) have a wealth of research on how to effectively evaluate and verify that the system has implemented the designed security controls [18-22]. Also, earlier stages (i.e. requirements) have received a great deal of attention from researchers over the last ten years. Security requirements identification, reasoning and evaluation have also been extensively researched [40-44]. Yet, very little has been done to evaluate the adherence of the design stage to the intended security requirement, in particular evaluating the software architecture. Open questions remain, such as how to evaluate the effectiveness of the integrated patterns to control specific security concerns as an aspect of the design stage in the framework, and how to assess the security of the architecture based on patterns. 


\section{REFERENCE}

[1] J. Viega and G. McGraw, Building Secure Software. Addison -Wesley, 2002.

[2] M. Howard, "Building more secure software with improved development process," IEEE Security \& Privacy, Vol.2, No.6, pp.63-65, 2004.

[3] N. Davis, W. Humphrey, S. R. Jr., G. Zibulski, and G. McGraw, "Processes for producing secure software," IEEE Security \& Privacy, Vol.2, No.3, pp.18-25, 2004.

[4] J. K. R and A. Mathur, "Software engineering for secure software - state of the art: A survey," tech. rep., Purdue University, 2005.

[5] A. Alkussayer and W. H. Allen, "Towards secure software development: Integrating security patterns into a secure SDLC," in The 47th ACM Southeast Conference, 2009.

[6] A. Alkussayer and W. Allen, "The ISDF framework: Integrating security patterns and best practices," in The 3rd International Conference on Information Security and Assurance (ISA'09), 2009.

[7] M. Howard and S. Lipner, The Security Development Lifecycle SDL: A Process for Developing Demonstrably More Secure Software. Microsoft Press, 2006.

[8] G. McGraw, Software Security: Building Security In. Addison-Wesley, 2006.

[9] M. Howard and S. Lipner, "Inside the windows security push," IEEE Security \& Privacy, Vol.1, No.1, pp.57-61, 2003.

[10] OWASP, “Comprehensive, lightweight application security process," http://www.owasp.org, 2009.

[11] G. McGraw, “Software security,” IEEE Security \& Privacy, Vol.2, No.2, pp.80-83, 2004.

[12] S. Simpson, "Fundamental practices for secure software development: A guide to the most effective secure development practices in use today." http://www.safecode.org, 2008

[13] M. Schumacher, E. Frenandez-Buglioni, D. Hybertson, F. Buschmann, and P. Sommerlad, Security Patterns: Integrating Security and Systems Engineering. John Wiley \& Sons, 2006.

[14] C. Alexander, S. Ishikawa, M. Jacobson, I. Fiksdahl-King, and S. Angel, A Pattern Language: Towns- Buildings- Construction. Oxford University Press, 1977.

[15] E. Gamma, R. Helm, R. Johnson, and J. Vlissides, Design Patterns: Elements of Reusable ObjectOriented Software. Addison- Wesley Professional, 1995.

[16] J. Yoder and J. Barcalow, "Architecural patterns for enabling application security," in PLoP97 Conference, 1997.

[17] T. Heyman, K. Yskout, R. Scandariato, and W. Joosen, "An analysis of the security patterns landscape,” in 3rd International workshop on Software Engineering for Secure Systems, 2007.

[18] G. H. G. McGraw, Exploiting Software: How to Break Code. Addison-Wesley, 2004.

[19] T. G. D. Graham, Software Inspection. Addison-Wesley,1993.

[20] M. Howard and D. LeBlance, Writing Secure Code. Microsoft Press, 2003.

[21] B. Potter and G. McGraw, "Software security testing," Software Development, Vol.2, No.5, pp.81-85, 2005.

[22] B. Chess and G. McGraw, "Static analysis for security,”Software Development, Vol.2, No.6, pp.7679, 2004.

[23] J. Gregoire, K. Buyens, B. Win, R. Scandariato, and W. Joosen, "On the secure software development process: CLASP and SDL compared," in Third International Workshop on Software Engineering for Secure Systems(SESS'07), 2007.

[24] C. Steel, R. Nagappan, and R. Lai, Core Security Patterns: Best Practices and Strategies forJ2EE, Web Services, and Identity Management. Prentice Hall Ptr, 2005.

[25] E. B. Fernandez and R. Pan,"A pattern language for security models," in PLoP 2001Conference, 2001.

[26] D. Hatebur, M. Heisel, and H. Schmidt, "Security engineering using problem frames," in International Conference on Emerging Trends in Information and Communication Security (ETRICS), 2006.

[27] M. A. Jackson, Problem Frames: Analysing and Structuring Software Development Problems. Addi- 
son-Wesley, 2001.

[28] V. Horvath and T. Dorges, "From security patterns to implementation using petri nets," in International Conference on Software Engineering, 2008.

[29] K. Supaporn, N. Prompoon, and T. Rojkangsadan, "An approach: Constructing the grammar from security patterns," in the 4th International Joint Conference on Computer Science and Software Engineering (JCSSE2007), 2007.

[30] K. Yskout, T. Heyman, R. Scandariato, and W. Joosen, “An inventory of security patterns,” tech. rep., Katholieke University Leuven, Department of Computer Science, 2006.

[31] M. Ha_z, P. Adamczyk, and R. Johnson, "Organizing security patterns,” IEEE Software, Vol.24, No.4, pp.52-60, 2007.

[32] B. Blakley, C. Heath, and M. of the Open Group Security Forum, "Security design patterns," tech. rep., Open Group, 2004.

[33] N. Yoshioka, H. Washizaki, and K. Maruyama, “A survey on security patterns,” Progress In Informatics, No.5, pp.35-47, 2008.

[34] M. Andrews and J. A. Whittaker, How to Break Web Software. Addison-Wesley, 2006.

[35] A. Aprville and M. Pourzandi, "Secure software development by example," IEEE Security \& Privacy, Vol.3, No.4, pp.10-17, 2005.

[36] J. Jurjens, Secure System Development with UML. Springer, 2004.

[37] I. Valenzuela, "Integration ISO17799 into your software development lifecycle," (In)Secure, Vol.11, pp.29-36, 2007.

[38] E. B. Fernandez, "A methodology for secure software design,” in International Conference on Software Engineering Research and Practice, 2004.

[39] E. B. Fernandez, N. Yoshioka, H. Washizaki, and J. Jurjens, "Using security patterns to build secure systems," in 1st International Workshop on Software Patterns and Quality (SPAQu'07), 2007.

[40] C. Haley, J. Mo_ett, R. Laney, and B. Nuseibeh, "A framework for security requirements engineering," in SESS'06, 2006.

[41] G. Sindre and A. Opdahl, "Eliciting security requirements with misuse cases," Requirements Engineering, Vol.10, No.1, pp.34-44, 2005.

[42] C. Haley, R. Laney, J. Mo_ett, and B. Nuseibeh, "The effect of trust assumptions on the elaboration of security requirements,” in Proceedings of the Requirements Engineering Conference, 12th IEEE International, 2004.

[43] N. Mead, E. Hough, and T. Stehney, "Security quality requirements engineering (SQUARE) methodology,” tech. rep., CMU/SEI-2005-TR-009, 2005.

[44] C. Haley, R. Laney, J. Mo_ett, and B. Nuseibeh, "Security requirements engineering: A framework for representation and analysis,” IEEE Transactions on Software Engineering, Vol.34, No.1, pp.133153, 2008.

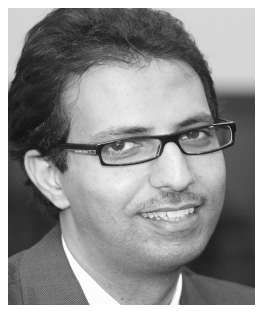

\section{Abdulaziz Alkussayer}

He received BS degrees in Information Systems from King Saud University, Saudi Arabia, in 1999, and an MS degree in Computer Science (with distinction) from California State University-Chico, USA, in 2004. He is currently working towards a PhD in the Department of Computer Science, Florida Institute of Technology-USA. His research interests include secure software development, security patterns, and security architecture evaluation. 


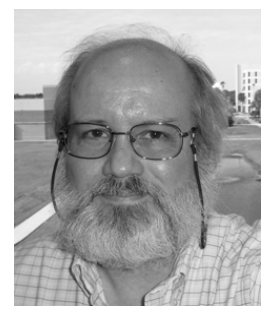

\section{William H. Allen}

Dr. William H. Allen is an Assistant Professor in the Department of Computer Sciences at the Florida Institute of Technology. His research interests include computer and information security, the modeling and simulation of network-based attacks, and computer forensics. Allen earned a PhD in Computer Science from the University of Central Florida. He currently supervises Master's and PhD students who are performing research in security policy, secure software development and automated attack generation for software testing and intrusion detection. 